\title{
Vom Fachkräftemangel in Ostdeutschland zum Comeback des Flächentarifvertrages? Ein Praxisbericht aus Sachsen-Anhalt
}

\author{
Norbert Kuck \\ Hartmut Meine \\ Thomas Müller
}

\begin{abstract}
Die Situation in den ostdeutschen Bundesländern ist seit mehr als 15 Jahren von Massenarbeitslosigkeit und einem deutlich niedrigeren Entgeltniveau als in Westdeutschland geprägt. Auch die Tarifbindung und der Stellenwert des Flächentarifvertrages sind im Osten Deutschlands deutlich geringer. Aktuell allerdings zeichnet sich eine erstaunliche Wende ab. Denn im Zuge der demografischen Entwicklung kommt es in Ostdeutschland bereits jetzt - anders als in Westdeutschland - zu einem spürbaren Fachkräftemangel. Das verschiebt die Angebots- und Nachfragesituation auf dem Arbeitsmarkt zugunsten der Beschäftigten und erhöht den Stellenwert des Flächentarifvertrages, was die Erklärungen der Tarifvertragsparteien und der Landesregierungen in mehreren Bundesländern deutlich zeigen.
\end{abstract}

\section{AUSGANGSSITUATION SEIT DER DEUTSCHEN EINHEIT}

Vor 20 Jahren, Anfang 1991, wurden in der Metallindustrie wie auch in anderen Branchen im Rahmen eines Stufenplanes die Flächentarifverträge aus Westdeutschland in Ostdeutschland übernommen. Dieser Prozess fand zunächst bei allen Beteiligten eine hohe Akzeptanz. Doch spätestens nachdem der massive De-Industrialisierungsprozess in Ostdeutschland zu einer dramatischen Massenarbeitslosigkeit geführt hatte, kam es zu einer Destabilisierung der neu übertragenen Flächentarifverträge. Die Relation von Angebot und Nachfrage auf den Arbeitsmärkten verschob sich zulasten der Arbeitnehmerseite. Viele Beschäftige in Ostdeutschland sahen sich angesichts der drohenden Arbeitslosigkeit gezwungen, Arbeit zu Entgeltbedingungen zu akzeptieren, die weit unter dem Niveau des Flächentarifvertrages lagen. Dadurch entwickelte sich in Ostdeutschland ein bedeutender Niedriglohnbereich.

In der Metallindustrie kündigten 1993 die Metallarbeitgeberverbände rechtswidrig den vereinbarten Stufenplan für die Flächentarifverträge. Der daraufhin entfachte Arbeitskampf mündete in einer Vereinbarung, den Stufenplan zu strecken und sogenannte Härteklauseln vorzusehen, die es ermöglichten, einzelbetriebliche Abwei- chungen vom Flächentarifvertrag durch die Tarifvertragsparteien zu vereinbaren. Doch trotz dieser „kontrollierten Flexibilisierung des Flächentarifvertrages" kam es zu einer weiteren Erosion des Tarifvertragssystems. Zahlreiche Unternehmen traten aus den tarifgebundenen Arbeitgeberverbänden aus. Die Arbeitgeberverbände gründeten sogenannte OT-Verbände (OT = ohne Tarif). Neu angesiedelte Firmen traten überwiegend nicht den Arbeitgeberverbänden bei. Und selbst in den tarifgebundenen Arbeitgeberverbänden war eine Radikalisierung zu beobachten, an deren Spitze sich die sächsischen Metallarbeitgeber stellten.

Diese Entwicklung verschärfte sich 2003 nach dem misslungenen Versuch der IG Metall, mit einem Streik die 35-Stunden-Woche auch in Ostdeutschland durchzusetzen. Aus der Perspektive vieler Arbeitgeber, der Politik, teilweise der Wissenschaft und der Medien galt das in Westdeutschland etablierte System der Flächentarifverträge nunmehr als für Ostdeutschland nicht kompatibel. Und selbst Teile der Arbeitgeberverbände stellten es jetzt generell infrage. Vielfach wurde die These vertreten, dass die Erosion des Systems der Flächentarifverträge zu einem neuen Regulierungsmodell führen würde, das stärker betriebliche bzw. individuelle Regelungen anstelle von tariflichen bein- halten würde. Auf diese Weise versprachen sich die Arbeitgeber, dauerhaft ein Entgeltniveau in Ostdeutschland durchzusetzen, das deutlich unter den Flächentarifverträgen liegt. Zahlreiche Firmen haben ihr Geschäftsmodell auf einem derartigen Niedriglohnmodell aufgebaut. Auch 20 Jahre nach der deutschen Einheit existieren - trotz formeller Angleichung der tariflichen Entgelte an Westdeutschland - bei den effektiv gezahlten Entgelten erhebliche Differenzen. Je nach Region und Branche betragen die tatsächlich gezahlten Effektiventgelte ca. 80 \% des Westniveaus. In vielen Branchen ist die Mehrheit der Arbeitsverhältnisse im Niedriglohnbereich und damit deutlich unter den für Ostdeutschland vereinbarten Flächentarifverträgen angesiedelt.

Norbert Kuck ist Bezirkssekretär in der IG
Metall Bezirksleitung Niedersachsen und
Sachsen-Anhalt.
e-mail: Norbert.Kuck@igmetall.de
Hartmut Meine ist Bezirksleiter des IG
Metall Bezirkes Niedersachsen und Sachsen-
Anhalt.
e-mail: Hartmut.Meine@igmetall.de
Thomas Müller ist Bezirkssekretär in der IG
Metall Bezirksleitung Niedersachsen und
Sachsen-Anhalt.
e-mail: Thomas.Mueller@igmetall.de


Das ist auch darauf zurückzuführen, dass in Ostdeutschland bis heute die Tarifbindung wesentlich niedriger ist als in Westdeutschland. So wurden beispielsweise $200956 \%$ der westdeutschen Beschäftigten von Tarifverträgen erfasst, dagegen nur $38 \%$ der ostdeutschen. Für das verarbeitende Gewerbe liegt dieser Wert in Westdeutschland bei $58 \%$ und in Ostdeutschland bei lediglich $25 \%$. $^{1}$

Angesichts der immer noch doppelt so hohen Arbeitslosigkeit wie in Westdeutschland und des niedrigen Entgeltniveaus ist es nicht verwunderlich, dass der Saldo von $\mathrm{Zu}$ und Abwanderung in Ostdeutschland stark negativ ist und zahlreiche Beschäftigte aus Ostdeutschland in nahe gelegene Regionen nach Westdeutschland pendeln. Ähnliche Entwicklungen sind bei der Abwanderung von Hochschulabsolventen sichtbar. Die inzwischen gut ausgebaute Hochschulstruktur in Ostdeutschland bietet hervorragende Ausbildungschancen, insbesondere im Bereich der Ingenieurwissenschaften und der sogenannten Mint-Fächer (Mint = Mathematik, Informatik, Naturwissenschaften, Technik). Die in Ostdeutschland ausgebildeten Ingenieure und Wissenschaftler werden aber massiv von westdeutschen Firmen umworben. Zahlreiche Absolventen der Ingenieurwissenschaften haben bereits vor Abschluss des Diploms Arbeitsverträge mit westdeutschen Firmen abgeschlossen.

In Sachsen-Anhalt wandern 75-80 \% der Absolventen eines ingenieurwissenschaftlichen Studiums unmittelbar nach Studienende nach Westdeutschland ab. ${ }^{2}$ Die Versorgung der ostdeutschen Industrie mit Fachkräften und insbesondere Ingenieuren ist damit schon aktuell und angesichts der sichtbaren Trends auch künftig gefährdet.

\section{ERWERBSCHANCEN UND -BEDIN- GUNGEN IN SACHSEN-ANHALT}

Die seit Anfang der 1990er Jahre desolate Arbeitsmarktsituation in Ostdeutschland prägte über Jahre hinweg auch die Situation in Sachsen-Anhalt. 15 Jahre lang betrugen selbst die offiziellen Arbeitslosenquoten hier oftmals $20 \%$ und mehr. Ohne den zeitweise riesigen zweiten Arbeitsmarkt und die Beschäftigungsgesellschaften, die häufig in engem Zusammenwirken mit den Gewerkschaften organisiert wurden, hätten die Arbeitslosenquoten bei $30 \%$ und mehr gelegen. Folglich galt auch hier: Durchsetzung von Niedriglöhnen auf breiter Front und ein bis heute andauernder
Abwanderungsstrom vor allem junger, gut qualifizierter Menschen. Dazu kommt ein großer Teil von Menschen, die gezwungen sind, lange Wege zur Arbeit in benachbarte westliche Bundesländer hinzunehmen. Bei 750.000 Beschäftigten in Sachsen-Anhalt pendeln weitere 140.000 Sachsen-Anhalter in andere Bundesländer. Der sogenannte Wanderungsverlust seit Herbst 1990 beträgt rund 260.000 Personen und umfasst $75 \%$ derjenigen im Alter von 15-49 Jahren. Zusammen mit der Geburtenentwicklung verlor das Land Sachsen-Anhalt seit 1989 fast $22 \%$ seiner Bevölkerung. ${ }^{3}$ Doch trotz dieser massiven „Entlastung“ des Arbeitsmarktes durch Abwanderung, Pendler und demografische Effekte blieb der Arbeitsmarkt bis heute von Massenarbeitslosigkeit geprägt. Die Arbeitslosenquoten lagen bis in die jüngste Vergangenheit hinein fast doppelt so hoch wie in Niedersachsen.

Hierfür mitverantwortlich waren zwei für Ostdeutschland spezifische Besonderheiten: Der massive Beschäftigungsabbau wurde in der ersten Hälfte der 1990er Jahre soweit wie möglich über Vorruhestandsmodelle abgefedert. Allerdings schieden über Jahre hinweg nur verhältnismäßig gering besetzte Jahrgänge aus den Betrieben aus. Zeitgleich aber suchten sehr starke Jahrgänge von Schulabgängern einen Einstieg ins Berufsleben. Dies war eine Folge der sehr erfolgreichen Familien- bzw. Geburtenförderung in der DDR in den 1970er Jahren, ${ }^{4}$ was unter den Arbeitsmarktverhältnissen in Ostdeutschland seit Mitte der 1990er Jahre in weiten Bereichen zu einem fast blockierten Berufseinstieg für junge Menschen führte. Für diese geburtenstarken Jahrgänge war der klassische Weg über die duale Ausbildung in einen Beruf viele Jahre fast versperrt. Die Länder und der Bund antworteten auf die Situation mit diversen Sonderprogrammen zur Schaffung von außerbetrieblichen Ausbildungsplätzen. Deren Anteil lag in den neuen Ländern bis in die jüngste Zeit weit über der Quote im Westen Deutschlands mit den bekannten Problemen beim Übergang an der sogenannten zweiten Schwelle. Noch 2006 fanden nur $39 \%$ eines Bewerberjahrgangs eine betriebliche Ausbildungsstelle, $7,4 \%$ wurden auf Plätze im Rahmen von Sonderprogrammen vermittelt, $10 \%$ besuchten zunächst weiter eine Schule und 5,5\% absolvierten vorerst ein Berufsvorbereitungsjahr oder ähnliche Maßnahmen. ${ }^{5}$ Folglich stieg auch der Anteil der sogenannten Altbewerber, also der Jugendlichen, die bereits in den Vorjahren eine Stelle gesucht hatten, auf rund $50 \% .2010$ stammt von den rund 14.000 jungen Menschen, die über die Bundesagentur für Arbeit einen Ausbildungsplatz suchen, rund die Hälfte aus dem Kreis der Altbewerber. Diese dramatische Situation auf dem Ausbildungsmarkt führte dazu, dass viele Unternehmen Ausbildungsvergütungen zahlten, die weit unterhalb des Niveaus des Flächentarifvertrages z.B. in der Metallindustrie lagen. ${ }^{6}$

\section{DEMOGRAFISCHE WENDE}

Inzwischen aber hat sich Entscheidendes verändert. Die Zahl der Schulabgänger ist - wie seit Langem von der Kultusministerkonferenz vorhergesagt - innerhalb weniger Jahre als Spätfolge des Geburtenknicks nach 1990 geradezu abgestürzt: von über 30.000 auf unter $15.000 \mathrm{im}$ Jahr 2010. Besonders starke Rückgänge gab es vor allem bei den Realschülern, von denen traditionell eine hohe Quote ins duale Ausbildungssystem wechselt. Diese Entwicklung ist völlig anders als z.B. im benachbarten Niedersachsen, wo ein moderater Rückgang der Schulabgängerzahlen erst nach 2015 einsetzt (Abbildung 1). Im aktuellen Ausbildungsjahr gibt es in Sachsen-Anhalt wie im Jahr 2009 mehr betriebliche Ausbildungsstellen als Bewerber aus dem aktuellen Schuljahr.

Gleichzeitig kommen in Sachsen-Anhalt seit 2005 wieder stärkere Jahrgänge von Älteren ins Rentenalter. Innerhalb weniger Jahre kippt so der Arbeitsmarkt von einem Ungleichgewicht ins andere. ${ }^{7}$ Die aus-

IAB Aktuell vom 29.03.2010.

2 Magdeburger Volksstimme vom 2.10.2008.

3 Statistisches Landesamt Sachsen-Anhalt (Hrsg.) (2010): Statistische Monatshefte Sachsen-Anhalt 08.

$4 \quad$ Erst mit dem Zusammenbruch der DDR kam es dann zu dem inzwischen viel beschriebenen Geburtenknick in Ostdeutschland, der die Geburten pro Frau an das westdeutsche Niveau anglich.

5 Vgl. Der Landtag von Sachsen-Anhalt (2007): Berufsbildungsbericht 2006 für das Land SachsenAnhalt, Drucksache 5/727.

6 Der Flächentarifvertrag sieht ca. $700 €$ im ersten Ausbildungsjahr vor. Im Gegensatz dazu wurden in außerbetrieblichen Ausbildungsringen 2007 wie beispielsweise im Ausbildungsprogramm Ost auf der Basis von Gerichtsurteilen lediglich $185 €$ (erstes Ausbildungsjahr) bis $215 €$ (viertes Ausbildungsjahr) gezahlt, in von der Bundesagentur für Arbeit geförderten außerbetrieblichen Ausbildungen 280 bis $325 €$.

7 Vgl. dazu Lutz, B. unter Mitarbeit von Grünert, H./Ketzmerick, T./Wiekert, J. (2010): Fachkräftemangel in Ostdeutschland, OBS-Arbeitsheft 65, Frankfurt/M. 
scheidenden älteren Beschäftigten können durch die nachrückenden jüngeren Jahrgänge in Zukunft nicht mehr ansatzweise ersetzt werden (Abbildung 2). Zwar verfügt der Arbeitsmarkt in Sachsen-Anhalt mit Blick auf rückkehrwillige Pendler und die immer noch zehntausenden Arbeitslosen weiterhin über ein Arbeitskräftepotenzial; jedoch müssten erst die Voraussetzungen geschaffen werden, um beide Gruppen auch tatsächlich in den Arbeitsmarkt SachsenAnhalts integrieren zu können. Für die Gewinnung von Pendlern dürfte der Abbau der Verdienstunterschiede entscheidend sein. Unter den Arbeitslosen wiederum fehlt es häufig an den benötigten Qualifikationen. ${ }^{8}$

Obwohl dieser massive Umschwung am Arbeitsmarkt seit Jahren absehbar gewesen ist, erfolgte das notwendige Gegensteuern eher zaghaft. Und viele Betriebe nahmen die Zeitenwende überhaupt nicht wahr. Noch 2006 vertrat einer Umfrage zufolge ${ }^{9}$ mehr als die Hälfte der Betriebe die Ansicht, dass die Zahl der Bewerber um Ausbildungsplätze bei ihrem Betrieb in Zukunft nicht zurückgehen werde, was die Forscher zu dem Fazit veranlasste, das Verhalten eines Großteils der Betriebe sei in gefährlichem Maße „vergangenheitsgeprägt“, die „Lernund Innovationsbereitschaft" gering.

\section{TARIFPOLITISCHE INITIATIVEN DER IG METALL}

Angesichts der skizzierten demografischen Entwicklung hatte die IG Metall bereits in der Tarifrunde 2007 den perspektivischen Fachkräftemangel thematisiert. Neben der Erhöhung der Entgelte um 6,5 \% forderte die IG Metall Bezirksleitung für Niedersachsen und Sachsen-Anhalt in der Tarifrunde drei Punkte:

- mehr betriebliche Ausbildungsplätze;

- mehr Studierende im Praxisverbund (Dualstudierende);

- mehr Stipendien für junge Ingenieure („Stipendientarifvertrag für Ingenieure“).

Die Reaktion des Verbandes der Metallund Elektroindustrie Sachsen-Anhalt war verhalten positiv: Einerseits schätzte er das Problem ähnlich wie die IG Metall ein. Andererseits sah sich der Arbeitgeberverband nicht in der Lage, dazu tarifliche Regelungen zu vereinbaren. Man verständigte sich darauf, weiter im Dialog zu bleiben. Daraus gingen im Oktober 2007 zwei gemeinsame Thesenpapiere zur Fachkräfteentwicklung
Abb. 1: Schulabgänger in Niedersachsen und Sachsen-Anhalt

- Messzahlen: $1995=100-$

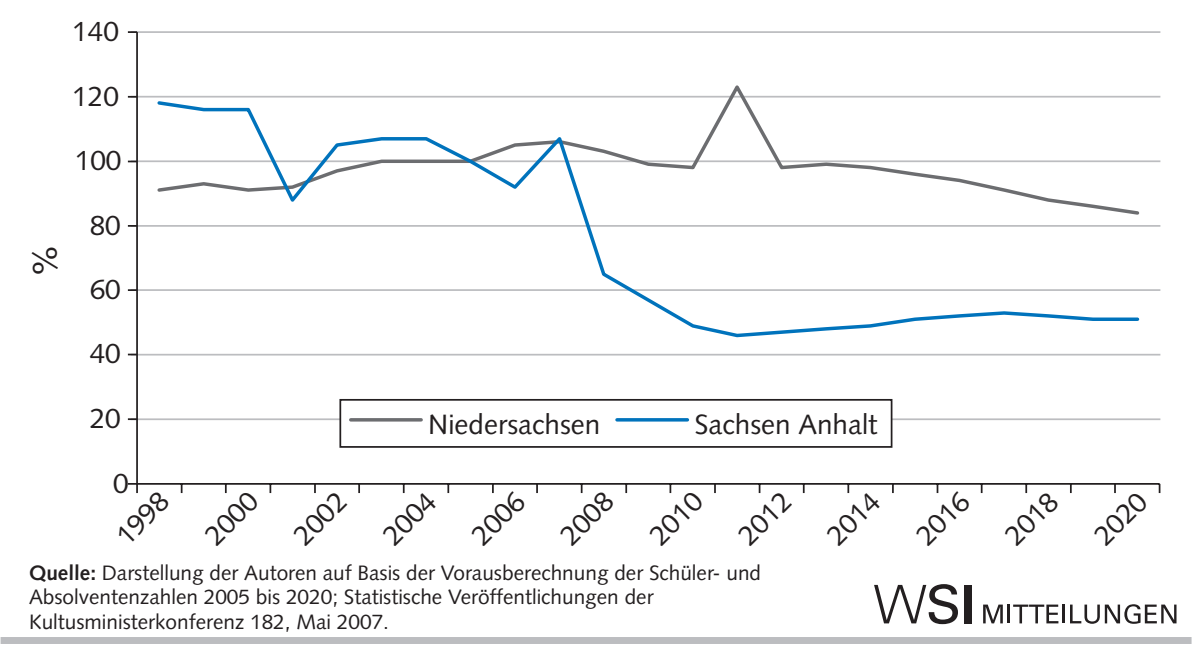

Kultusministerkonferenz 182, Mai 2007.

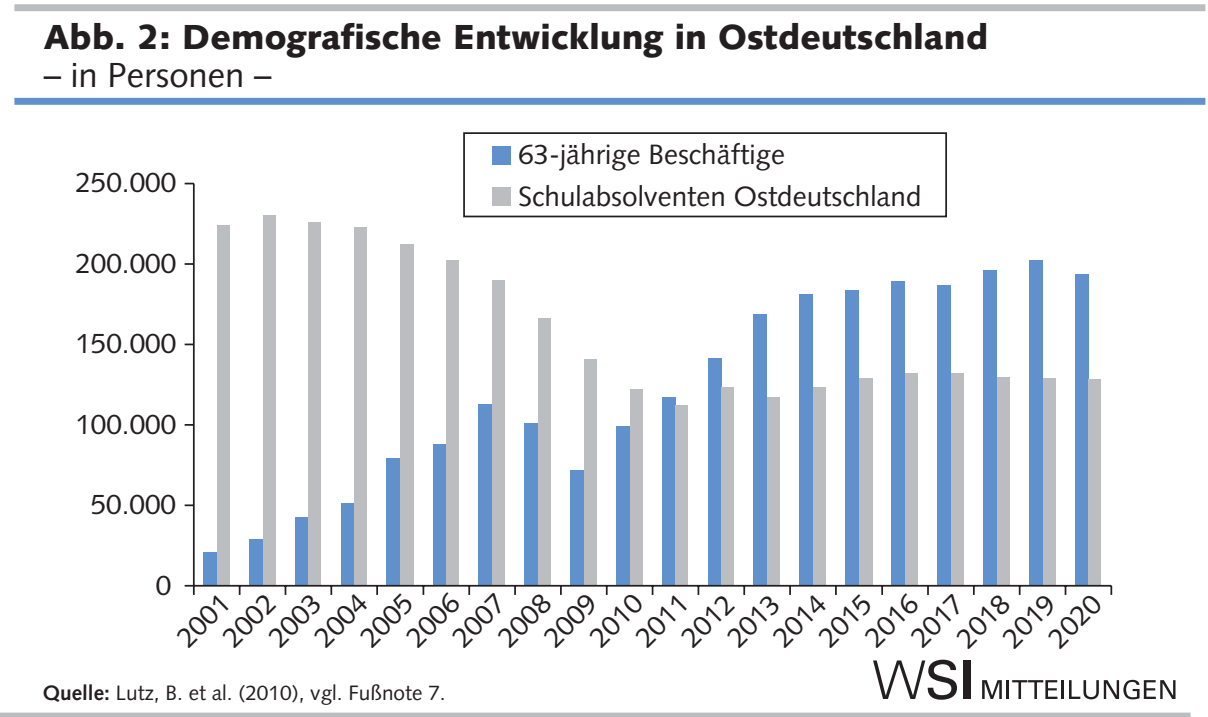

in Sachsen-Anhalt hervor. Geteilt wurden die Einschätzungen, dass ,partielle Schwierigkeiten bei der Stellenbesetzung von Ingenieuren, aber auch bei hochqualifizierten Fachkräften wie Zerspanungsmechanikern (CNC-Fachkräften), Mechatronikern, Industriemechanikern und Schweißern" ${ }^{10}$ existieren und dass die Schaffung zusätzlicher Ausbildungsstellen ein Weg sein kann, um dem sich abzeichnenden Fachkräftemangel entgegenzuwirken.

Da die Wirtschaftskrise in den Jahren 2008 und 2009 in Ostdeutschland weniger spürbar war als in Westdeutschland, blieb auch in den Krisenjahren das Thema Fachkräftemangel aktuell und erhielt mit Anziehen der Konjunktur eine gewisse Brisanz. Für die Metall- und Elektroindustrie gilt aktuell, dass es insbesondere in Ballungsräumen um Magdeburg, aber auch im Wirtschaftsraum Halle/Bitterfeld, bereits nachweisbar Schwierigkeiten gibt, Fachkräfte zu finden.

Nicht viel anders stellt sich die Situation im Feld der beruflichen Ausbildung

8 Vgl. Ministerium für Wirtschaft und Arbeit des Landes Sachsen-Anhalt (2009): Analyse der Fachkräftesituation im Land Sachsen-Anhalt und Ausblick bis 2016, 16.12., Magdeburg.

9 Vgl. dazu Grünert, H./Lutz, B./Wiekert, I. (2007): Betriebliche Ausbildung und Arbeitsmarktlage - eine vergleichende Untersuchung in Sachsen-Anhalt, Brandenburg und Niedersachsen, Forschungsberichte aus dem zsh 5, Zentrum für Sozialforschung Halle.

10 Verband der Metall- und Elektroindustrie Sachsen-Anhalt e.V./IG Metall Bezirksleitung Niedersachsen und Sachsen-Anhalt: Thesen zur Fachkräfteentwicklung in der Metall- und Elektroindustrie in Sachsen-Anhalt, Oktober 2007 
dar. Durch die drastische Reduzierung der Schulabgängerzahlen hat sich das Verhältnis von Angebot und Nachfrage auf dem Ausbildungsstellenmarkt deutlich zugunsten der Bewerberinnen und Bewerber gewandelt. Vor diesem Hintergrund startete die IG Metall 2008 eine Aktion, die sich an Schulabgänger richtete. Unter dem Motto „Du bist mehr wert!“ wurden Schülerinnen und Schüler durch Informationsmaterialien darauf hingewiesen, dass sie es nicht mehr nötig haben, ein Ausbildungsverhältnis einzugehen, das nicht den Bedingungen des Flächentarifvertrages entspricht. Ihnen wurde empfohlen, bei der Bewerbung um einen Ausbildungsplatz gezielt nachzufragen, ob der Betrieb tarifgebunden ist. Daraufhin sahen sich zahlreiche Betriebe gezwungen, die Ausbildungsvergütungen zu erhöhen und am Niveau des Flächentarifvertrages zu orientieren. Aufgrund des $\$ 17$ Abs. 1 des Berufbildungsgesetzes und eines entsprechenden Urteils des Bundesarbeitsgerichtes müssen auch in nicht-tarifgebundenen Betrieben mindestens $80 \%$ der Ausbildungsvergütungen des jeweiligen Tarifvertrages gezahlt werden. Mit Unterstützung der IG Metall haben zahlreiche Auszubildende in nicht-tarifgebundenen Betrieben dieses Niveau erfolgreich vor den Arbeitsgerichten geltend gemacht. Innerhalb weniger Jahre hat sich eine deutliche Verschiebung der Ausbildungsvergütungen in der Metallindustrie in Richtung der Vorgaben des Flächentarifvertrags abgezeichnet.

\section{TARIFPOLITISCHE SCHLUSS- FOLGERUNGEN}

Die demografische Entwicklung - insbesondere das Ausscheiden älterer Jahrgänge und die wesentlich geringere Zahl von Schulabgängern sowie die weiter bestehenden Abwanderungstendenzen - hat trotz hoher Massenarbeitslosigkeit in Teilsegmenten der Facharbeit zu einer Verschiebung von Angebot und Nachfrage zugunsten der Arbeitnehmerseite geführt. Allein nach den Marktgesetzen ergibt sich aufgrund der Knappheit von Fachkräften eine tendenzielle Erhöhung des Entgeltniveaus. Wenn einzelne Fachkräfte deswegen in der Lage sind, auszuwählen, in welchem Betrieb sie arbeiten, führt dies zu einer tendenziellen Erhöhung des Entgeltniveaus. Fachkräfte können nur gehalten und geworben werden, wenn entsprechend höhere Entgelte gezahlt werden. Aufgrund des spürbar werdenden Fachkräftemangels erkennen mehr und mehr Unternehmen in der Metallindustrie und in anderen Branchen, dass ihr auf Niedriglöhnen basierendes Geschäftsmodell nicht mehr tragfähig ist. Diese absehbare Entwicklung ist eingetreten und hat in Teilsegmenten des Arbeitsmarktes tatsächlich schon jetzt zu entsprechend höheren Entgelten geführt. Im Zuge dessen hat sich eine relevante Zahl von Betrieben der Metall- und Elektroindustrie, die vor Jahren aus dem tarifgebundenen Arbeitgeberverband ausgetreten sind, entschlossen, in den Arbeitgeberverband zurückzukehren und die Flächentarifverträge anzuwenden. Teilweise haben die IG Metall und der Arbeitgeberverband dazu Heranführungsregelungen vereinbart. Diese Entwicklung ist kein Automatismus, bietet aber für die IG Metall bessere Chancen als in der Vergangenheit, die Tarifbindung und damit das Entgeltniveau zu erhöhen. In diese Richtung weisen auch weitere Initiativen, die in Sachsen-Anhalt stattgefunden haben.

\section{ERKLÄRUNG ZUR TARIF- PARTNERSCHAFT}

Der Fachkräftemangel hat in SachsenAnhalt verschiedene Akteure auf den Plan gerufen. So wurde beispielsweise unter Federführung der CDU/SPD-geführten Landesregierung am 24. Juni 2010 ein Fachkräftesicherungspakt abgeschlossen, in dem eine Strategie zur Fachkräftesicherung für die Wirtschaft im Land Sachsen-Anhalt definiert wurde. Neben der Landesregierung haben der Deutsche Gewerkschaftsbund, die Dachvereinigung der Arbeitgeber- und Wirtschaftsverbände, die Industrie- und Handelskammern, die Handwerkskammern sowie die Regionaldirektion der Bundesagentur für Arbeit diese Vereinbarung unterzeichnet. Auf Drängen der Gewerkschaften wurde in den Fachkräftesicherungspakt folgende Passage aufgenommen: „Durch attraktive Entgelt- und Arbeitsbedingungen können Fachkräfte gehalten und geworben werden. Die Aufgabe der Tarifvertragsparteien ist es, durch attraktive Flächentarifverträge die Tarifbindung zu erhöhen ".11

Weiterhin wurde auf Initiative der Gewerkschaften verabredet, dass eine separate Vereinbarung zur Tarifpartnerschaft in Sachsen-Anhalt abgeschlossen werden sollte. Eine vergleichbare Erklärung wurde bereits am 30. März 2010 in Thüringen zwischen dem Minister für Wirtschaft, Arbeit und Technologie sowie den Gewerkschaften und den Arbeitgeberverbänden mehrerer Industriebranchen vereinbart. Nach längeren intensiven Debatten konnte am 21. Oktober 2010 auch in Sachsen-Anhalt eine „Erklärung zur Stärkung der Tarifpartnerschaft" vereinbart werden. ${ }^{12}$ Unterzeichnet wurde diese vom CDU-Wirtschaftsminister, auf der Gewerkschaftsseite von der IG Metall, der IG BCE, der IG BAU und der NGG sowie auf Arbeitgeberseite von den tarifgebundenen Arbeitgeberverbänden der Metall-, Holz- und Chemieindustrie sowie dem Bauindustrieverband, dem Baugewerbeverband und dem Verband der Ernährungswirtschaft. In dieser Erklärung wurde das Ziel einer deutlichen Erhöhung der Tarifbindung formuliert. So heißt es etwa: „Der Minister für Wirtschaft und Arbeit und die beteiligten Tarifparteien sind sich in diesem Zusammenhang einig, dass in Tarifverträgen geregelte Entgelt- und Arbeitsbedingungen eine wichtige Grundlage für die Wirtschaft darstellen, um im Wettbewerb um knapper werdende Fachkräfte und Auszubildende erfolgreich zu sein. Damit stellt die Anwendung der Flächentarifverträge ein Gütezeichen für eine zukunftsorientierte Personalpolitik in den Unternehmen dar“. Weiter heißt es in der Erklärung: „Auf dieser Basis setzen sich die Unterzeichner für eine stärkere Beteiligung der bisher nicht organisierten Unternehmen und Beschäftigten ein und treten für starke Arbeitgeberverbände und Gewerkschaften ein (...). Das Ministerium für Wirtschaft und Arbeit wird bei Kontakten mit Unternehmen auf die Bedeutung der Flächentarifverträge und die Mitgliedschaft bei den Tarifpartnern hinweisen. Dies geschieht auch bei der Vergabe öffentlicher Fördergelder" ${ }^{\text {"13 }}$

Mit den Erklärungen zur Erhöhung der Tarifbindung in Thüringen und SachsenAnhalt haben sich wichtige Akteure in Politik und Wirtschaft eindeutig für eine Stärkung des Flächentarifvertrages ausgesprochen. Bedenkt man die Aggressivität, mit der gerade in Ostdeutschland gegen das System des Flächentarifvertrages in der Öffentlichkeit argumentiert wurde, kann ohne Übertreibung von einem Paradigmenwechsel gesprochen werden. Unsere These lautet: In der ostdeutschen Industrie steht der Flächentarifvertrag vor einem Comeback.

11 Fachkräftesicherungspakt 2010. Strategie zur Fachkräftesicherung für die Wirtschaft im Land Sachsen-Anhalt, Magdeburg 24.06.

12 Vgl. http://www.igmetall-nieder-sachsen-anhalt. de/News-Details.25+M5e9e43e55db.0.html

$13 \mathrm{Ebd}$ 